\title{
TENDENCIAS DE LA INVESTIGACIÓN SOBRE REINTEGRACIÓN Y REINCORPORACIÓN DE EXCOMBATIENTES EN COLOMBIA. TENSIONES Y OPORTUNIDADES
}

\author{
Javier Alonso Cárdenas-Díaz \\ DANiela Tocarruncho-HernÁNDEZ ${ }^{* *}$ \\ Aura Lerma-Zambrano ${ }^{* * *}$
}

\section{Resumen}

El objetivo de este artículo es presentar los patrones y las tendencias en el proceso de apoyo de la Agencia para la Reincorporación y la Normalización (ARN) a las propuestas de investigaciones de la comunidad científica sobre reintegración y reincorporación de excombatientes en Colombia. La ARN ha sido, durante 15 años, la entidad del Estado encargada de apoyar a los excombatientes de grupos armados ilegales a su reintegración y reincorporación a la vida civil en Colombia. En este camino se ha reconocido que la construcción de redes de conocimiento con otras entidades y actores es fundamental para fortalecer los procesos de diseño, implementación, evaluación y mejora de las políticas públicas a cargo de la ARN. Ello produjo que desde el año 2008 hasta 2019 se estudiaran formalmente 305 propuestas de investigaciones de universidades, investigadores, profesores y estudiantes tanto nacionales como de otros países. De ellas, 160 han contado con el apoyo de la ARN. Esta investigación muestra los principales actores que se dedican a la investigación (entre universidades internacionales

\footnotetext{
* Doctor en Ciencia Política. Maestría en ciencia política. Investigador líder del Grupo de Investigación "Construcción de paz, desarme, desmovilización, reintegración y reincorporación” (Colombia). [ja.cardenas909@uniandes.edu.co], [ORCID: https://orcid.org/0000-0002-1414-3833].

" Politóloga. Investigadora del Grupo de Investigación "Construcción de paz, desarme, desmovilización, reintegración y reincorporación” (ARN) (Colombia). [tocarrunchohernandezdaniela@gmail.com],

[ORCID: https://orcid.org/0000-0003-3027-8620].

${ }^{* *}$ Administradora pública. Estudiante de la Especialización en Planeación, gestión y control del desarrollo social. Investigadora del Grupo de Investigación "Construcción de paz, desarme, desmovilización, reintegración y reincorporación (Colombia). [aura.lermaz@gmail.com], [orcid: https://orcid.org/0000-0002-0674-2377].

Recibido: 15 de septiembre de 2019 / Modificado: 14 de enero de 2020 / Aceptado: 13 de febrero de 2020

Para citar este artículo

Cárdenas-Díaz, J. A., Tocarruncho-Hernández, D. y Lerma-Zambrano, A. (2020). Tendencias de la investigación sobre reintegración y reincorporación de excombatientes en Colombia. Tensiones y oportunidades. OPERA, 27, 119-140 DoI: https://doi.org/10.18601/16578651.n27.06
} 
y nacionales), los grados de especialización en los estudios, los temas más relevantes, así como las metodologías más utilizadas en los estudios sobre reintegración y reincorporación de excombatientes. Para ello se utiliza una metodología cuantitativa de carácter descriptivo y longitudinal sobre la base de datos de apoyo a investigaciones externas de la ARN, y se resaltan aspectos relevantes en profundidad mediante el análisis cualitativo de entrevistas realizadas a funcionarios nacionales y regionales de la ARN.

Palabras clave: ciencia, gobernanza, investigación, paz, reintegración, reincorporación.

\section{RESEARCH TRENDS ON REINTEGRATION AND REINCORPORATION OF EX-COMBATANTS IN COLOMBIA.TENSIONS AND OPPORTUNITIES}

\section{Abstract}

The purpose of this article is to present the patterns and trends of the Agency for Reincorporation and Normalization (ARN) in supporting research proposals from the scientific community on reintegration and reincorporation of ex-combatants in Colombia. For the last 15 years, the ARN has been the governmental institution in charge of supporting reintegration and reincorporation of illegal armed groups' ex-combatants to civilian life. In doing so, it has been acknowledged that building knowledge networks with other institutions and actors is a fundamental task in order to strengthen the design, implementation, evaluation and improvement processes of public policies of the ARN. This is why between 2008 and 2019, 305 research proposals by universi- ties, researchers, professors and students were studied from both Colombia and abroad. One hundred sixty of these were supported by the ARN. This research shows the main actors that do research on this topic (including international and national universities), the degree of specialization in these studies, the most relevant topics, and the most used methodologies in studies on reintegration and reincorporation of ex-combatants. To do this, we use a descriptive and longitudinal quantitative methodology on the data base of the supported research proposals by the ARN, and highlight the relevant aspects by conducting qualitative analysis from interviews with national and regional officials of the ARN.

Key words: Science; governance; research; peace; reintegration; reincorporation.

\section{INTRODUCCIÓN}

La Agencia para la Reincorporación y la Normalización (ARN) ha sido, durante 15 años, la institución del Estado en Colombia encargada de apoyar a los exintegrantes de grupos armados ilegales a su retorno a la vida civil ${ }^{1}$.

1 Los protocolos y las rutas de reintegración y reincorporación de excombatientes han sido construidos desde el año 2003, momento en que el Ministerio del Interior asumió el Programa de Reinserción a la Vida Civil (PRVC). Este programa fue evolucionando hasta convertirse en una Alta Consejería en el ańo 2006, para posteriormente constituirse en una Unidad Administrativa Especial en 2011 con el nombre de Agencia Colombiana para la Reintegración (ACR). Producto de los Acuerdos de Paz con las FARC-EP, la organización cambió de nombre a Agencia para la Reincorporación y la Normalización (ARN), y a pesar de asumir esta nueva denominación, 
En este camino, se ha reconocido que la construcción de redes de conocimiento con otras organizaciones y actores es fundamental para fortalecer los procesos de diseño, implementación, evaluación y mejora de las políticas públicas a cargo de la ARN. Ejemplo de ello fue la celebración del Congreso Internacional de Desarme, Desmovilización y Reintegración (CIDDR) en el año 2009, del cual se produjo como resultado el documento guía Contribución de Cartagena al desarme, la desmovilización y la reintegración (2009), o el Congreso en Santa Marta del desarme, la desmovilización y la reintegración (DDR) llevado a cabo en el año 2014 (Cárdenas, 2015), eventos que convocaron a expertos internacionales, profesionales y líderes de programas del DDR para compartir experiencias y conocimientos, así como elaborar documentos guía para fortalecer elementos de la reintegración de excombatientes.

Además de estos espacios de cooperación técnica, y para promover la generación y transferencia de conocimiento, la ARN ha diseñado herramientas a fin de orientar y apoyar a los investigadores e instituciones dedicadas a la ciencia interesados en investigar sobre la reintegración de excombatientes. Estas herramientas obedecen a dos necesidades. La primera, promover la convergencia entre los intereses de las organizaciones y actores científicos, y los objetivos de la reintegración. La segunda,

la ruta de reintegración se mantuvo para atender a los excombatientes que se desmovilizan individualmente, y la experiencia ha servido como insumo para diseńar la ruta de reincorporación colectiva de los antiguos miembros de las FARC-EP. Para conocer mejor cómo la ARN ha respondido a las modificaciones institucionales producto del Acuerdo de Paz con las FARC-EP, ver Cárdenas (2019, pp. 69-85). y considerando la condición de vulnerabilidad presente en los actores que han participado en el conflicto armado en Colombia, evitar posibles perjuicios y garantizar el cuidado de la población que participa en los proyectos de ciencia e investigación, estableciendo unos criterios mínimos que deben cumplir estos proyectos.

Al cumplir 10 años de haber abierto las puertas a los actores e instituciones científicas, y de haber estudiado formalmente 305 proyectos de investigación, se inició un proceso de reflexión sobre los patrones y las tendencias que se han dado en la investigación sobre la reintegración y reincorporación de excombatientes en Colombia, con base en una metodología cuantitativa de carácter descriptivo y longitudinal. Los resultados de este ejercicio son presentados en este documento con el objeto de analizar, desde la óptica de la política pública, las convergencias y las tensiones entre la investigación científica y la atención a población vulnerable en el marco de la violencia y el conflicto en Colombia, particularmente en el proceso de reintegración de excombatientes ${ }^{2}$.

Ante un posible aumento de la producción científica como producto de la firma del Acuerdo de Paz realizado en 2016 entre el Gobierno colombiano y las Fuerzas Armadas Revolucionarias de Colombia-Ejército del Pueblo (FARC-EP), este documento espera aportar elementos que contribuyan a materializar una gobernanza de la ciencia, entendida esta como el conjunto de reglas, intereses, inte-

2 Se entiende por reintegración de excombatientes el proceso en el que estos son orientados por planes de atención psicosocial, adquieren un estatus civil e ingresos sustentables (CCDDR, 2009). 
racciones y recursos entre el Gobierno y los actores de la innovación y la ciencia con el fin de resolver problemas o crear oportunidades sociales (Kooiman, 2003; Bora y Hausendorf, 2006; Kulhmann, 2007), en donde los científicos y las instituciones del Gobierno trabajen articuladamente para construir conocimiento en torno a la reintegración y reincorporación de excombatientes en Colombia.

\section{GOBERNANZA, CIENCIA Y SOCIEDAD}

Según Bora y Hausendorf (2006), el concepto de Gobernanza fue originalmente asociado al análisis económico, definido como un medio de regulación y control político, legal y social que es ejercido principalmente sobre las corporaciones, pero cuyo sentido se puede extender más allá de lo mercantil (Amin, 1997). De acuerdo con lo expuesto por Baunen y Opdam (2011), este concepto se ha ido transformando, tradicionalmente se le daba prioridad al sector político y empresarial, en una relación donde el científico funcionaba principalmente como un asesor para los responsables políticos. Actualmente, este modelo se ha inclinado hacia la coordinación y fusión de lo público, lo privado y la ciudadanía, pues ahora todos los anteriores resultan ser actores con acción participativa. Teniendo en cuenta lo anterior, Kooiman (2003) define gobernanza como:

La totalidad de interacciones, en las que participan actores tanto públicos como privados, destinados a resolver problemas sociales o crear oportunidades sociales; considerando las instituciones como contextos para estas interacciones de gobernanza; y estableciendo una base normativa para todas estas actividades. (p. 4)
Siguiendo con esta línea argumentativa, algunos autores como Lehoux et al. (2010) examinan las acciones de gobernanza, es decir, aquellas prácticas que tienen campo de acción en medio del sistema gubernamental, las cuales se encuentran contenidas en tres tipos principales de interacciones. El primer tipo de interacción es aquel que se presenta entre investigadores de diferentes disciplinas, las cuales ayudan a gestionar intercambios de conocimiento que involucran a distintas culturas epistémicas a través de las cuales se define lo que ha de ser investigado, esto conduce a una producción de conocimiento caracterizada por ser interdisciplinaria.

El segundo tipo se refiere a las interacciones entre investigadores y decision makers (actores con capacidad decisoria), que requieren de una adecuada gobernanza institucional, por medio de la cual se organizan, gestionan y ejecutan mecanismos que articulen y prioricen las investigaciones y los proyectos transdisciplinarios orientados por temáticas de trabajo.

Finalmente, se presenta la interacción entre las redes de cooperación científica y el entorno político, la cual tiene como base la producción de políticas u otros productos derivados de la investigación, que se encuentran orientados a ayudar en la toma de decisiones políticas. Knorr (1999) llama a estos productos de investigación "objetos epistémicos", dado que por medio de su circulación se transforma el entorno político gracias al cambio de las representaciones públicas que impactan posteriormente en las acciones de gobernanza.

Por otra parte, Bora y Hausendorf (2006) entienden gobernanza como una relación 
social cooperativa entre instituciones gubernamentales y actores de la sociedad civil, enfoque que difiere ligeramente de la definición de Kooiman al poner más énfasis en "relación social”, en lugar de "interacción", esto como consecuencia de lo teóricamente difícil que resulta usar el término interacción para definir tanto las relaciones entre pequeños actores como las que se desarrollan entre grandes organizaciones.

Regresando a Baunen y Opdam (2011), estos autores afirman que anteriormente el papel de la ciencia estaba atado a describir fenómenos genéricos y universales, que tienen que ser reinterpretados para ser aplicados en los entornos locales. Con base en lo anterior, sugieren la importancia de empezar a generar diálogos y redes de cooperación entre diferentes actores. De acuerdo con esto, Lyall (2007) señala que la gobernanza implica un cambio de la regulación estatal de asuntos económicos hasta cierto grado de autorregulación por grupos sociales responsables. Cooke (2002) acuña el término de "gobernanza asociativa" como un sistema en el que los organismos administrativos regionales son interactivos e inclusivos con respecto a otros actores regionales. Si bien gran parte de los sistemas tradicionales de innovación se enfocan sobre las interacciones que conducen al aprendizaje entre empresas, a menudo descuidan las interacciones entre los actores del sistema (empresas / base científica / intermediarios) y, por tanto, el proceso de aprendizaje entre las empresas sobre las interacciones entre el Gobierno y otros actores dentro del nivel central, que podría ayudar a informar el proceso de aprendizaje de políticas.
La interacción entre estos diferentes actores que menciona Lyall (2007) permite que se desarrollen redes politicas, las cuales constituyen un importante componente de la teoría de la gobernanza. Borzel (1998) ofrece una definición de red de políticas como un conjunto relativamente estable de relaciones que son de naturaleza no jerárquica e interdependiente, vincular una variedad de actores que comparten intereses comunes con respecto a una política, intercambiar recursos para perseguir estos intereses compartidos y, al hacerlo, reconocer que la cooperación es la mejor manera de alcanzar objetivos comunes; basado en esto, Lyall (2007) desarrolla el término "gobernanza multinivel”, la cual se desarrolló por primera vez como un marco conceptual para reconocer los roles interpretados por varios actores en el escenario de la política que, adicionalmente, conecta diferentes escenarios políticos.

Lo anterior está muy relacionado con el concepto de Geuna y Muscio (2009) de Gobierno de transferencia, que se refiere a la comercialización y transferencia del conocimiento, que se presenta en instituciones como las universidades, proceso a través del cual no solo se comparte el conocimiento con otros actores de la sociedad, sino que ayuda a fortalecer económicamente al sector que ofrece el servicio, lo que permite dinamizar todo el sector académico y científico.

Teniendo en cuenta este contexto, la ARN, en el marco de la Estrategia de Gestión del Conocimiento y la Innovación, ha diseñado herramientas para orientar y apoyar a los investigadores e instituciones dedicadas a la ciencia interesados en investigar sobre la reintegración de excombatientes. Con estas herramientas se 
busca, en primer lugar, promover la convergencia entre los intereses de las organizaciones y los actores científicos con los objetivos de la reintegración. Y, en segundo lugar, promover una gobernanza ética (Comisión Europea, 2009).

La gobernanza ética implica reconocer la condición de vulnerabilidad presente en los actores que han participado en el conflicto armado en Colombia, para así evitar posibles perjuicios y garantizar el cuidado de la población que participa en los proyectos de ciencia e investigación, estableciendo unos criterios mínimos que deben cumplir estos proyectos. De esta forma, la entidad busca contribuir a materializar una gobernanza de la ciencia, en donde los científicos y las instituciones de gobierno trabajen articuladamente para construir conocimiento.

\section{METODOLOGÍA}

Para comprender los resultados de este estudio, el lector debe tener en cuenta dos aspectos fundamentales: en primer lugar, los proyectos de ciencia e investigación analizados corresponden solamente a aquellos que han sido articulados con la ARN, en consecuencia, aquellos realizados de forma independiente no fueron incluidos en la muestra seleccionada.

En segundo lugar, en este estudio se decidió seleccionar los proyectos desarrollados durante el periodo 2008-2018, lo que implica que la mayoría de ellos están relacionados con el proceso de reintegración de excombatientes, el cual durante el periodo 2008-2016 fue el único proceso de la política del Estado colombiano para la atención y el apoyo a los exintegrantes de grupos armados al margen de la ley (GAOML). En este proceso han participado las personas que se desmovilizaron de forma colectiva pertenecientes a las Autodefensas Unidas de Colombia (AUC), y las personas que se han desmovilizado de forma individual de grupos como el Ejército de Liberación Nacional (ELN), el Ejército Popular de Liberación (EPL) y las FARC-EP.

En 2016, con la firma del acuerdo de paz entre el Gobierno colombiano y las FARC-EP, se empezó a diseñar un proceso para las personas de este grupo que dejaron las armas de manera colectiva, el cual fue denominado proceso de reincorporación ${ }^{3}$. De esta forma, inicia una nueva fase en la política de Estado para la atención de excombatientes en Colombia, que se viene desarrollando paralelamente con el proceso de reintegración. Por ende, se debe tener en cuenta que los primeros ańos que abarca este estudio, entre 2008 y 2016, obedecen a las tendencias de la investigación de los actores científicos sobre el proceso de reintegración implementado antes de los Acuerdos de La Habana. Los dos últimos años del análisis, por tanto, tienen en cuenta información del proceso de reintegración y del de reincorporación de las FARC-EP.

Otro factor que se debe tener en cuenta es que, según información suministrada por la ARN, durante 2016-2018 se han presentado novedades en la articulación con la comunidad científica para adelantar proyectos de investi-

3 El lector interesado en comprender las razones por las que se decidió utilizar el nombre de reincorporación puede consultar el texto "Los debates de La Habana: una mirada desde adentro", realizado por el Institute for Integrated Transitions (IFIT). 
gación sobre el proceso de reincorporación, debido a que es un proceso que se encuentra en fase de construcción. De hecho, hasta el 22 de junio de 2018 se logró institucionalizar la política pública para la reincorporación de excombatientes mediante el Conpes 3931. Esto implica que existen desafíos en la manera en que las instituciones científicas y sus actores puedan impactar en una política en proceso de formulación y diseńo. De esta forma, cuando el proceso cuente con mayores niveles de madurez organizacional se puede esperar un aumento en las interacciones con la comunidad científica para la producción de conocimiento en materia de reincorporación.

Ahora bien, los 305 proyectos en ciencia e investigación que desde 2008 hasta 2018 se han acercado a la ARN para crear conocimiento sobre asuntos de reintegración, reincorporación y paz, han sido registrados en una base de datos donde se consigna la información básica del proyecto, así como datos que dan cuenta de su contenido, tales como: título, área de conocimiento, tema, objetivo general y específico, aporte para la ARN, cobertura territorial, metodología e instrumentos metodológicos.

Es a partir de la base de datos de la ARN, que se hace seguimiento del proceso de cada proyecto que se desarrolla este artículo. Este análisis tomó como muestra aquellos 180 proyectos que la ARN aprobó bajo dos criterios: técnico y contenido sustancial. En el primero, se evalúa la coherencia, pertinencia y alcance entre el planteamiento del problema, la justificación, los objetivos, el marco teórico o conceptual, la metodología, el cronograma y los recursos que se emplearán para su desarrollo. En el segundo, se identifica la forma en la que la ARN puede contribuir al logro de sus objetivos, el tipo de apoyo (características y condiciones), y los insumos y posibles impactos que los resultados del proyecto podrían proporcionar a los procesos a cargo de la ARN.

Para abordar este estudio se optó por hacer uso de una metodología cuantitativa de carácter descriptivo y longitudinal, debido a que permitía caracterizar los datos registrados a lo largo del periodo de análisis (López, Roldán y Fachelli, 2015) y agruparlos a través de gráficos de líneas, columnas y áreas, buscando una representación directa de las frecuencias absolutas o porcentuales.

Con la base de datos de la ARN decidimos crear nuevas categorías para establecer las tendencias de las investigaciones en reintegración o reincorporación de excombatientes que obedecen a los siguientes asuntos: tema de la investigación, departamento de ejecución, metodologías e instrumentos metodológicos utilizados, instituciones de educación superior que avalan las investigaciones y áreas de conocimiento. Una vez compilados estos datos, se procedió a describir y analizar su contenido, con el objetivo de identificar las tendencias en cada categoría y las posibles relaciones entre las mismas.

Así, fue posible establecer la cantidad y el porcentaje de los temas que han sido abordados con mayor constancia en las regiones, la frecuencia temporal en temas, áreas de conocimiento, metodologías e instrumentos metodológicos. También, se comparó la frecuencia temporal de la participación de las instituciones de educación superior a nivel nacional e internacional que avalan los proyectos de investigación. 
Con el objetivo de profundizar en el análisis, se hizo uso de entrevistas semiestructuradas como herramienta de análisis cualitativo, aplicadas a siete funcionarios de la ARN encargados de evaluar los proyectos de ciencia e investigación que solicitan apoyo para su ejecución.

El contenido de la entrevista giró en torno a cuatro ejes: 1) aportes esperados y generados por la comunidad científica, 2) análisis del componente ético en los proyectos de ciencia e investigación, 3) temas sobrediagnosticados o subestudiados, 4) formulación de recomendaciones para la comunidad académica y científica que se interesa por asuntos de reintegración, reincorporación y paz. Nos interesaba saber si había, al respecto, coincidencia entre los valores de los resultados del análisis cuantitativo y las percepciones de estos funcionarios. Queríamos saber, además, algunos detalles de importancia que probablemente no pudieran ser capturados mediante las tendencias, como algunas dificultades o desafíos en el plano ético, así como su percepción sobre el impacto de estas investigaciones al interior de la ARN.

Paralelo a esto, se realizaron entrevistas vía telefónica a los funcionarios encargados de la coordinación de la política de la ARN en los territorios ubicados en Cundinamarca, Boyacá, Antioquia, Chocó, Valle del Cauca, Meta y Orinoquia. Escogimos estas regiones porque los resultados cuantitativos arrojaron, como se verá más adelante, que es en estas donde se implementan la mayoría de proyectos de ciencia e investigación en reintegración o reincorporación de excombatientes.

Estas entrevistas tenían por objetivo indagar sobre los temas abordados por las investigaciones así como aquellos que deberían serlo, los aportes a las instituciones locales, la identificación de dificultades, la pertinencia de las herramientas metodológicas y la generación de recomendaciones para contribuir a la creación de una verdadera gobernanza de la ciencia.

Una vez transcrito el contenido del total de las entrevistas realizadas, se construyó una matriz de forma tal que fue posible sistematizar e identificar patrones en las respuestas, para luego cotejarlas con los hallazgos del análisis cuantitativo. Nuestro propósito era comprender cómo interactuaban las tendencias en los temas, las instituciones de educación superior que avalan los proyectos de investigación y las metodologías y los instrumentos metodológicos utilizados con las perspectivas de los territorios. Los hallazgos cuantitativos y cualitativos del ejercicio y sus resultados serán presentados a continuación.

\section{ANÁLISIS}

\section{Tendencias históricas}

La primera investigación recibida y estudiada formalmente por la ARN -en ese entonces Alta Consejería Presidencial para la Reintegración ${ }^{4}$

4 La Agencia para la Reincorporación y la Normalización (ARN) ha tenido diferentes cambios administrativos y jurídicos desde su creación. Sus orígenes se remontan al Programa para la Reincorporación y la Vida Civil del Ministerio del Interior, creado en 2003. Luego, en septiembre de 2006 se creó la Alta Consejería Presidencial para la Reintegración con el fin de responder a las exigencias del proceso de desmovilización en Colombia -particularmente el aumento de personas que ingresaron con la desmovilización masiva de las Autodefensas Unidas de Colombia (AUC)-. Posteriormente, con el 
fue en el ańo 2008. En ese momento, y hasta 2012, la relación de la institución con la ciencia se basó en promover la visibilidad del proceso de reintegración de excombatientes, a través de la generación de productos académicos.

En el marco del proceso de apoyo a investigaciones externas, los primeros acercamientos entre la institución y la comunidad científica se basaron en la entrega de información. Sin embargo, con ocasión del fortalecimiento y la evolución del proceso de atención a la población excombatiente, generado por la expedición de la Política Nacional de Reintegración Social y Económica para personas y grupos armados ilegales (Conpes 3554 de 2008), la forma de abordar el tema desde la ciencia empezó a sufrir una transformación, la cual se evidencia en el aumento de las solicitudes para realizar entrevistas con la población beneficiaria del proceso de reintegración ${ }^{5}$.

Decreto 4138 de 2011, la Alta Consejería se convierte en una Unidad Administrativa Especial con personería jurídica, adscrita al Departamento Administrativo de la Presidencia de la República (DAPRE), bajo el nombre de Agencia Colombiana para la Reintegración. Finalmente, luego de la firma del Acuerdo Final para la terminación del conflicto realizado entre el Gobierno nacional y las FARC-EP, la entidad cambia su denominación y objeto con el Decreto Ley 897 del 29 de mayo de 2017 para responder a las necesidades del proceso de reincorporación; así las cosas, pasa a ser la ARN.

5 Las personas que acceden al proceso de reintegración son aquellas acreditadas como desmovilizadas por el Comité Operativo para la Dejación de las Armas (CODA) o la Oficina del Alto Comisionado para la Paz (OACP), que se desmovilizaron después del 24 de enero de 2003. En el proceso participan personas que fueron parte de las FARC -que se desmovilizaron individualmente-, Ejército de Liberación Nacional (ELN), Ejército Popular de Liberación (EPL) y las Autodefensas Unidas de Colombia (AUC).
En este nuevo escenario, la ARN se convirtió en un intermediario entre los investigadores y la población beneficiaria del proceso de reintegración, por lo cual se empezó a fortalecer la rigurosidad en la revisión y aprobación de las solicitudes de apoyo, para evitar que los instrumentos de recolección de información generaran posibles afectaciones a la población (psicológicas, legales o asociadas con su seguridad). Una vez revisadas y aprobadas las propuestas, se inicia el proceso de gestión y búsqueda de la población excombatiente que de manera voluntaria deseaba colaborar con la investigación.

Posteriormente, con la experiencia acumulada en los 5 años de análisis y aprobación de propuestas se crearon cinco instrumentos que permitieron fortalecer la rigurosidad del proceso. Primero, se creó un procedimiento para estudiar y hacer seguimiento a las solicitudes de apoyo por parte de instituciones de ciencia e investigación, así como actores externos, junto con una guía y un formato que contiene los criterios básicos que deben revisar los funcionarios encargados de estudiar las propuestas.

Los criterios básicos que debe contener un proyecto de ciencia e investigación fueron también incluidos en una guía disponible en la página web de la institución, en la cual se solicita describir, como mínimo: planteamiento del problema, justificación, objetivos, marco teórico o conceptual, metodología utilizada, así como el cronograma planeado. Asimismo, se solicita detallar de forma concreta y clara cuál es el apoyo que se requiere, así como anexar los instrumentos de recolección de información. 
Adicionalmente, se creó un documento denominado "Compromiso de confidencialidad", en el cual los investigadores se comprometen a tomar las medidas necesarias para proteger la naturaleza de la confidencialidad de la información, los documentos o el material suministrados por la ARN que tienen el carácter de reservados ${ }^{6}$, así como a transferir el conocimiento generado producto del apoyo realizado por la institución.

Esta última acción representó un cambio en el enfoque en la relación gobierno-ciencia, debido a que el interés primordial ya no era visibilizar el proceso de reintegración en el país, sino generar conocimiento para fortalecer los procesos de diseño, implementación, evaluación y mejora de las políticas públicas a cargo de la ARN, por lo cual la transferencia de conocimiento se convirtió en un elemento fundamental.

Como se observa en la figura 1, este cambio de enfoque incidió en el aumento de propuestas de ciencia e investigación rechazadas por la ARN. En 2013, el porcentaje de rechazos respecto al número de total de propuestas recibidas fue del $9 \%$, el cual fue aumentando progresivamente hasta representar el $73 \%$ para el año 2018. Se puede observar que desde el año 2015 se registra un cambio justo cuando la ARN implementa unos criterios estables de estudio sobre investigaciones y proyectos

6 Por encontrarse relacionados con la Defensa y Seguridad Nacional, de conformidad con lo dispuesto por el Capítulo II, artículo 24 de la Ley 1437 de 2011, por la cual se expide el Código de Procedimiento Administrativo y de lo Contencioso Administrativo, modificado por el artículo 1 de la Ley 1755 de 2015. de ciencia en reintegración. La línea negra, que simboliza el número de proyectos cuya solicitud de colaboración de la ARN fue rechazada, por primera vez supera la línea punteada que simboliza el número de investigaciones que recibieron la aprobación de la ARN. Más adelante se abordarán otros factores que explican las razones de esta importante variación.

En la figura 1 también se refleja el aumento considerable en las solicitudes de apoyo realizadas, lo cual ha generado un esfuerzo cada vez mayor por parte de la ARN, en especial, para las instituciones locales que han recibido más investigadores, entre las cuales se encuentran Bogotá y sus alrededores (Cundinamarca y Boyacá) con el $45 \%$ del total de investigaciones, seguido de Antioquia-Chocó con el 10\%, Valle del Cauca y Meta con el 6\% cada una, y finalmente Santander con el 4\%. Esto implicó para la ARN, de acuerdo con las entrevistas realizadas a funcionarios, poner a disposición un grupo de profesionales que tuvieran funciones permanentes, no solo de revisión de proyectos relacionados con la investigación en materia de reintegración, sino de seguimiento, y, en general, el contacto con los diferentes actores del sistema de ciencia.

\section{Áreas de conocimiento}

Al contar con la información de la base de datos de la ARN, queríamos saber si existía alguna tendencia sobre campos científicos particulares que pudiesen estar más interesados que otros en estudiar la reintegración, la reincorporación de excombatientes y la paz. Lo que se encontró es que áreas de conocimiento como la ciencia política, la psicología, la sociología, la economía 
FIGURA 1. INVESTIGACIONES QUE HAN SOLICITADO APOYO A LA ARN ENTRE 2008-2018

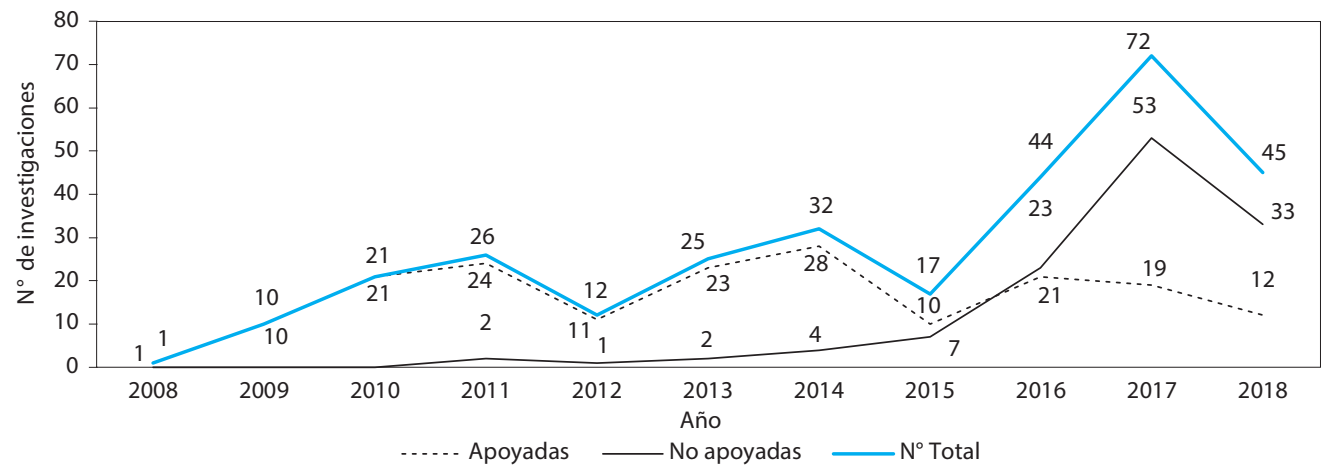

Fuente: elaboración propia con base en los datos de la Oficina Asesora de Planeación (ARN, 2019).

y la pedagogía son los ámbitos científicos más afines con los procesos que ejecuta la ARN. Los resultados pueden verse en la figura 2.

Estos resultados nos permiten sugerir que existe una potencial comunidad científica alrededor de los temas de paz, pero en específico acerca de los procesos de reintegración y reincorporación de excombatientes. Es posible establecer que hay, en este sentido, un marco de especialización e institucionalización de la ciencia. Se podría esperar, por ejemplo, que existiese una literatura académica y unos actores de ciencia e innovación dedicados exclusivamente a la reintegración y reincorporación de excombatientes. Esto implica también una suerte de formalización del campo científico. Queríamos, además, saber cómo habían sido las trayectorias en el tiempo de las investigaciones considerando estos campos especializados. Los resultados de las tendencias por área de conocimiento en el tiempo son presentados en la figura 3.
Como se puede observar, existe una cierta tendencia con pequeñas variaciones en el tiempo de la frecuencia de investigaciones en los campos de la ciencia política y la psicología. Las áreas de la sociología, la economía y la pedagogía, sin embargo, parecen ser muy sensibles a cambios en el tiempo, y parecen ser esporádicas en ciertos momentos, llegando incluso en algunos ańos a no recibir propuestas de investigación en estos campos de conocimiento.

\section{Temas}

A lo largo del periodo de análisis se han abordado 22 temas, dentro de los que se destacan: género, reintegración económica, percepciones sobre excombatientes y paz, reconciliaciónconvivencia, y niños, niñas, adolescentes y jóvenes (NNAJ). Contrario a temas como DDR, estigmatización, posconflicto y reincorporación, como se muestra en la figura 4. 
FIGURA 2. ÁREAS DE CONOCIMIENTO ABORDADAS EN LAS INVESTIGACIONES APOYADAS POR LA ARN ENTRE 2008-2018

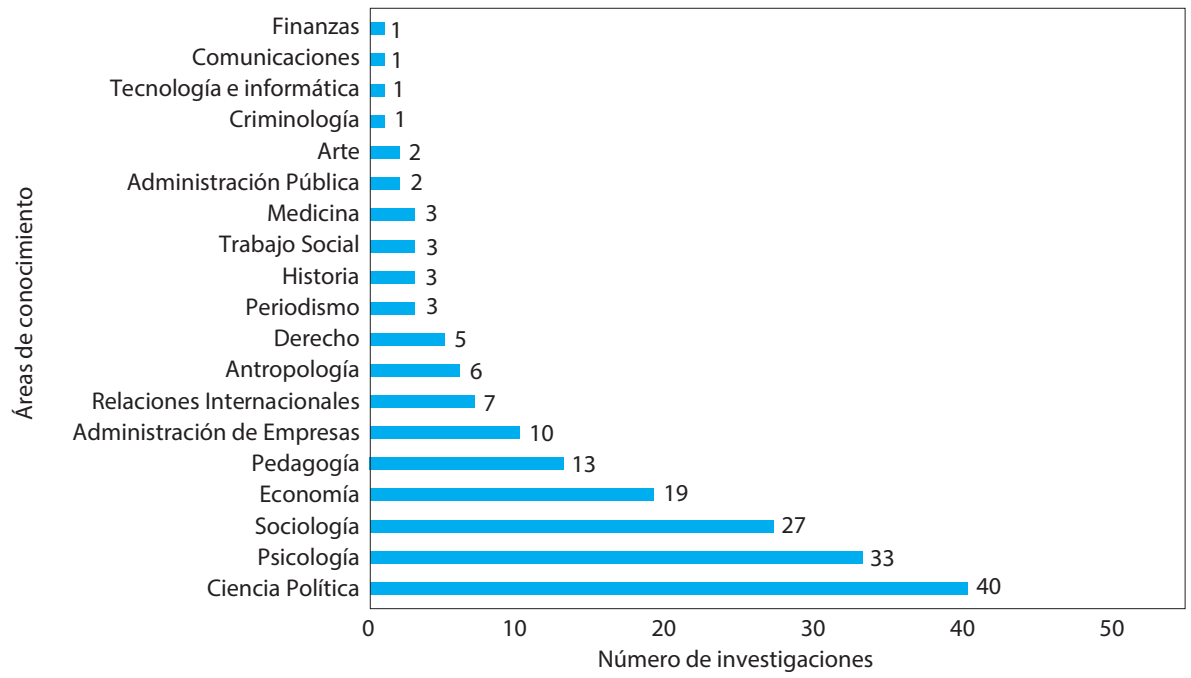

Fuente: elaboración propia con base en los datos de la Oficina Asesora de Planeación (ARN, 2019).

\section{FIGURA 3. TENDENCIAS POR ÁREA DE CONOCIMIENTO DE LAS INVESTIGACIONES APOYADAS POR LA ARN ENTRE 2008-2018}

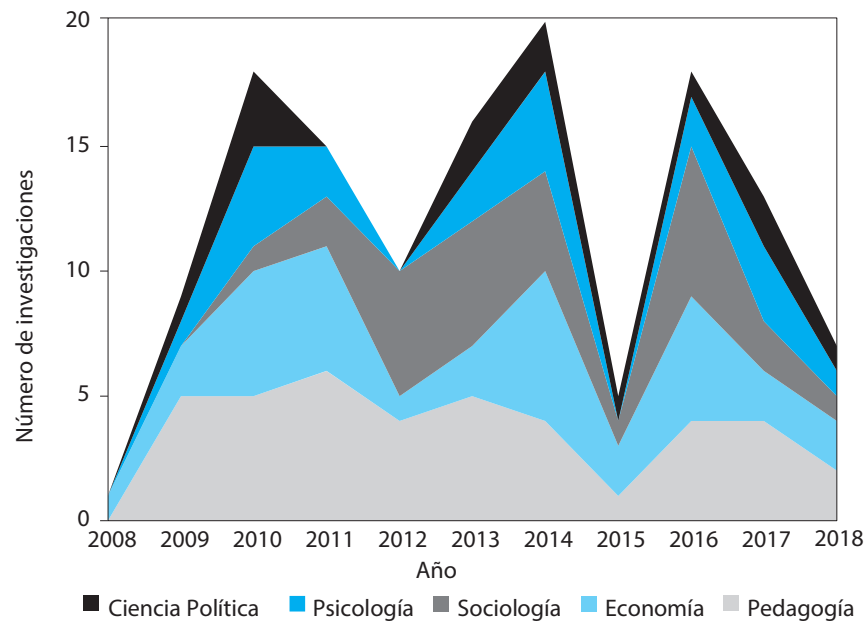

Fuente: elaboración propia con base en los datos de la Oficina Asesora de Planeación (ARN, 2019). 


\section{FIGURA 4. TEMAS ABORDADOS}

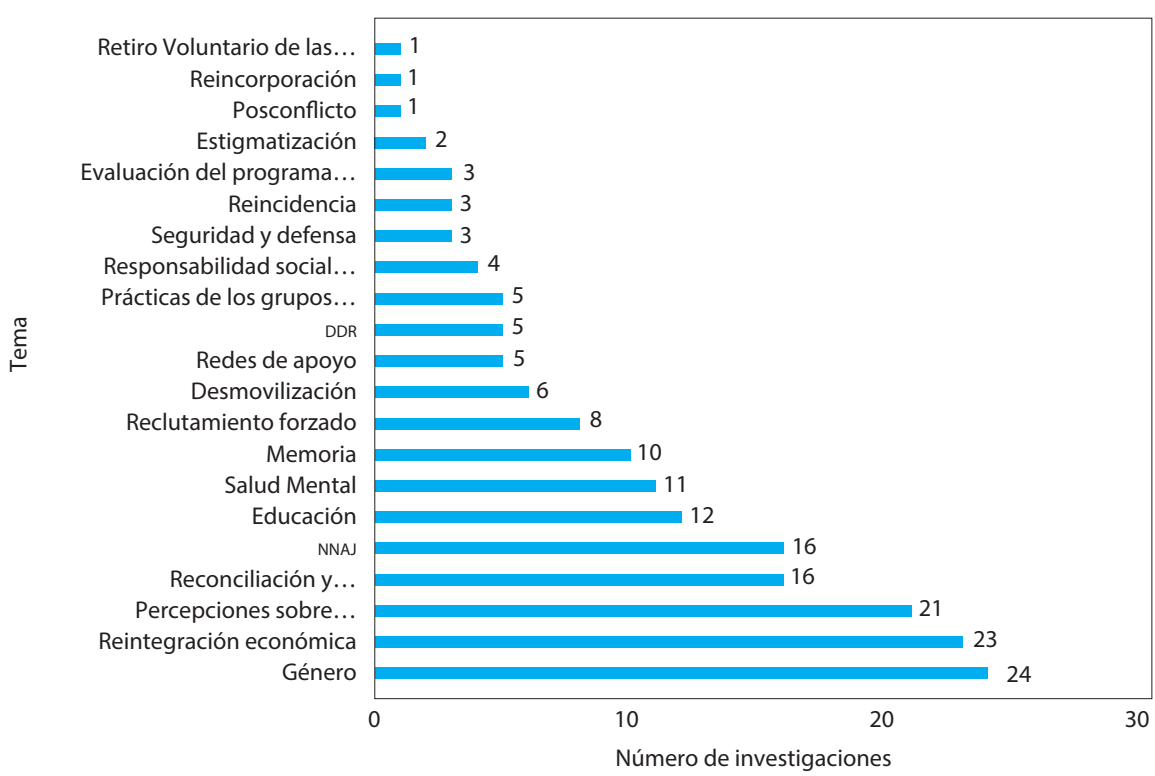

Fuente: elaboración propia con base en los datos de la Oficina Asesora de Planeación (ARN, 2019).

Queríamos saber cómo había sido el comportamiento histórico de los principales temas estudiados en materia de reintegración, reincorporación y paz. Con miras a reconocer la frecuencia de abordaje de los principales temas durante el periodo de análisis se construyó la gráfica de la figura 5, donde los temas reconciliación-convivencia y NNAJ presentaron alzas en los años 2011 y entre 2013 y 2014; mientras que género, reintegración económica y percepciones sobre excombatientes y paz fueron desarrollados de manera constante y representaron el $38 \%$ de la muestra total.

Debido a que estos temas han sido los de mayor interés para la comunidad académica y científica, se decidió profundizar en el análisis de contenido de cada uno de los proyectos que abordaron estos temas, logrando así la identificación de características específicas presentadas a continuación.

El tema de género fue abordado en 24 proyectos, que desarrollaron su trabajo de campo en las regiones de Cundinamarca-Boyacá, Antioquia, Valle del Cauca, Atlántico, Eje Cafetero, Santander, Tolima, Meta-Orinoquia. En estas se indagó por las relaciones de género o los roles de la mujer dentro de los grupos armados al margen de la ley, las maternidades en el posconflicto, los obstáculos y las oportunidades en la inserción laboral, el rol de la mujer excombatiente en la construcción de la paz, los procesos de reintegración de las mujeres, las transformaciones de las identidades de género de excombatientes y las artes como una 
FIGURA 5. FRECUENCIA DE LOS PRINCIPALES TEMAS ENTRE 2008-2018

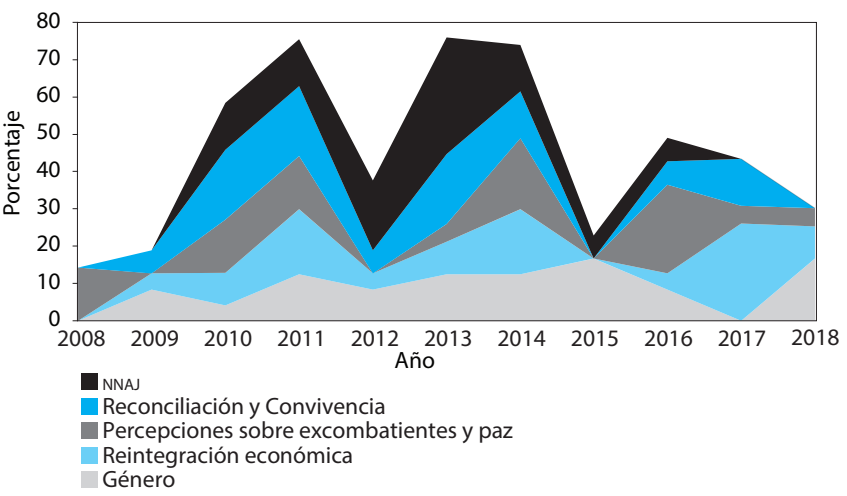

Fuente: elaboración propia con base en los datos de la Oficina Asesora de Planeación (ARN, 2019).

oportunidad para transformar las huellas de la violencia en las mujeres.

Durante las entrevistas aplicadas, los funcionarios destacaron aquellas investigaciones que contribuyeron a la visibilización y comprensión de fenómenos que podrían estar influyendo positiva o negativamente en los avances de la inclusión del enfoque de género en los programas de la ARN, además de socializar herramientas metodológicas para su ejecución.

Frente al tema de reintegración económica ${ }^{7}$ desarrollado en 23 proyectos de investigación, se analizaron fenómenos que dan cuenta de los obstáculos y las oportunidades del tránsito al

7 El Conpes 3554 de 2008, especifica dos de los objetivos estratégicos de la reintegración económica: 1) contribuir a la generación de capacidades, destrezas y habilidades en la población desmovilizada que les permita, como a cualquier colombiano, desempeńarse adecuadamente en el mercado laboral; 2) facilitar el ingreso a la actividad económica legal generando habilidades laborales, apoyando proceso de empleabilidad y facilitando y desarrollando planes productivos. mercado laboral, la responsabilidad empresarial, la incidencia del proceso de reintegración en la generación de ingresos, el mejoramiento de la calidad de vida gracias a la empleabilidad y el impacto de las iniciativas de emprendimiento. Proyectos ejecutados en las regiones de Cundinamarca-Boyacá, Antioquia, Córdoba, Cauca, Santander, Meta-Orinoquia; teniendo alzas en los años 2011, 2014, 2017 y 2018.

En lo que concierne al tema percepciones sobre excombatientes y paz, la ARN apoyó 21 investigaciones, ejecutadas en las regiones de Cundinamarca-Boyacá, Antioquia, Santander, Eje Cafetero, Atlántico, Huila, Nariño y Valle del Cauca, desarrolladas en mayor número en los años 2011, 2014 y 2016. Estas se enfocaron en acercarse a las narrativas, los imaginarios y las justificaciones presentes en diferentes grupos sociales y territoriales frente a lo que significa la reincorporación de los excombatientes, la construcción de paz en los territorios, los discursos del Gobierno nacional y la otredad en el posconflicto. Teniendo como un objetivo común la comprensión de los límites y las 
condiciones sociales que favorecen la inclusión de los excombatientes a la vida civil.

Frente a este tema, los funcionarios manifestaron que la ausencia de retroalimentaciones impedía conocer los aportes fundamentales del desarrollo de estas investigaciones para los programas o proyectos de la ARN, lo que a su vez genera inconformidad por parte de las personas que participan voluntariamente como sujetos de investigación, quienes manifiestan dudas sobre cómo su participación aporta al buen desarrollo de su proceso de reintegración o reincorporación.

La anterior caracterización de los principales temas abordados en las investigaciones apoyadas, y las afirmaciones de los funcionarios entrevistados, hacen posible identificar vacíos de conocimiento en temas como: estigmatización, evaluación de los programas de la ARN, reincidencia (refiriéndose a los factores que impulsan a excombatientes a retornar a las vías de hecho), enfoques diferenciales, entre otros, que abrirían discusiones necesarias para avanzar de manera conjunta en la planificación y ejecución de acciones concretas para los procesos de reincorporación.

\section{Actores de ciencia e investigación que impulsan las investigaciones en reintegración, reincorporación y paz}

Como escenarios de construcción de conocimiento, las instituciones de estudios superiores juegan un rol fundamental en los procesos de reintegración, y ahora reincorporación de los excombatientes; sus aportes a través de la reflexión teórica y práctica desde diferentes disciplinas y contextos sociales permiten nutrir las políticas y los programas enfocados hacia la paz. En el caso de las investigaciones apoyadas por la ARN, el $61 \%$ ha contado con el amparo de universidades nacionales, teniendo una participación significativa en los años 2011, 2013, 2014 y 2016. El 39\% restante corresponde a universidades extranjeras, que desde 2009 han sido constantes en el periodo de tiempo analizado, teniendo un alza significativa en el año 2014 (figura 6); de estas se destacan instituciones de países como Estados Unidos, Alemania, Inglaterra y Francia.

Las investigaciones desarrolladas entre 2009 y 2018, con el amparo de universidades extranjeras, han abordado con mayor frecuencia temas de género, NNAJ, reconciliación-convivencia y reintegración económica; el 59\% de estas han sido ejecutadas en CundinamarcaBoyacá, seguidas por Antioquia, Norte de Santander, Tolima y Valle del Cauca. En lo que respecta al $61 \%$ de las investigaciones amparadas por instituciones de educación superior colombianas, los temas destacados han sido género, percepciones sobre excombatientes y paz, reconciliación-convivencia y salud mental. De estas, el $91 \%$ han sido desarrolladas por universidades privadas, destacándose la Pontificia Universidad Javeriana, Universidad de los Andes, Universidad del Rosario, Universidad Externado de Colombia y la Universidad del Norte. La participación de universidades públicas corresponde al $9 \%$ del total, destacándose la Universidad Nacional de Colombia, la Universidad del Cauca y la Universidad Pedagógica Nacional.

A lo largo de las entrevistas realizadas a los funcionarios de la ARN, estos aseguraron que el aporte fundamental de las investigaciones 
FIGURA 6. FRECUENCIA DE UNIVERSIDADES NACIONALES FRENTE A UNIVERSIDADES EXTRAJERAS

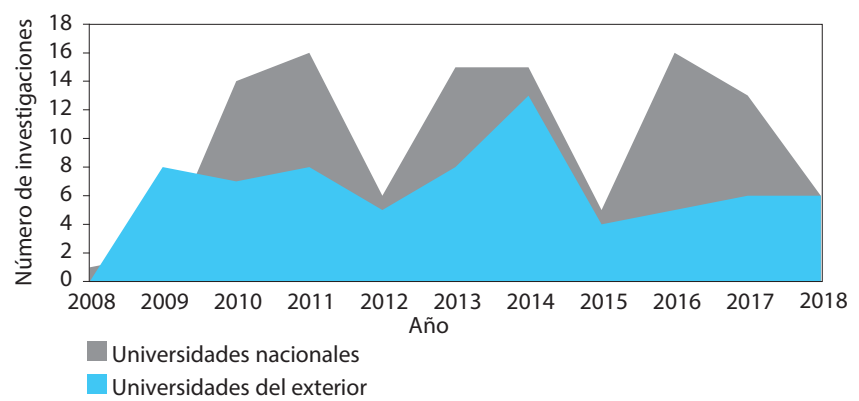

Fuente: elaboración propia con base en los datos de la Oficina Asesora de Planeación (ARN, 2019).

amparadas por universidades del exterior giraba en torno a la socialización de los programas de construcción de paz liderados por la entidad en contextos con características similares y diferentes. Sin embargo, consideran que sus aportes a los programas y procesos de la ARN son limitados, a causa de factores que giran en torno al tipo de conocimiento que adquieren para el planteamiento de las investigaciones, al reducido tiempo del trabajo de campo, a las brechas culturales y lingüísticas que se hacen presentes en la aplicación de las herramientas metodológicas, entre otros.

Frente a las instituciones nacionales, aseguran que es de suma importancia hacer un llamado a aquellas que se ubican en las regiones del país, para que desarrollen investigaciones a largo plazo, haciendo uso del conocimiento tácito y explícito que han adquirido como sujetos inmersos en el contexto. Lo anterior debido a que los aportes de investigaciones con dicha característica han abierto paso a la comprensión de fenómenos invisibilizados, sus causas y consecuencias, así como las posibles vías para iniciar o mejorar acciones en pro de la construcción conjunta de soluciones.

\section{Enfoques metodológicos}

Otro de nuestros intereses era conocer con qué enfoques y perspectivas metodológicas, y por ende epistemológicas, los actores en ciencia e investigación abordaban los problemas de estudio sobre reintegración y reincorporación, así como la paz. Lo que muestra la figura 7 es que una gran mayoría de investigaciones son formuladas bajo enfoques cualitativos de investigación. Casi el $80 \%$ de las investigaciones en reintegración y reincorporación utilizan estrategias de investigación cualitativas. Por el contrario, las investigaciones cuantitativas han sido relativamente pocas (14). Un resultado favorable para la diversidad de los enfoques es que 25 investigaciones han combinado estrategias cualitativas y cuantitativas. Sin embargo, creemos que se deben aprovechar mejor los datos cuantitativos en materia de reintegración y reincorporación de excombatientes. 
FIGURA 7. ENFOQUES METODOLÓGICOS

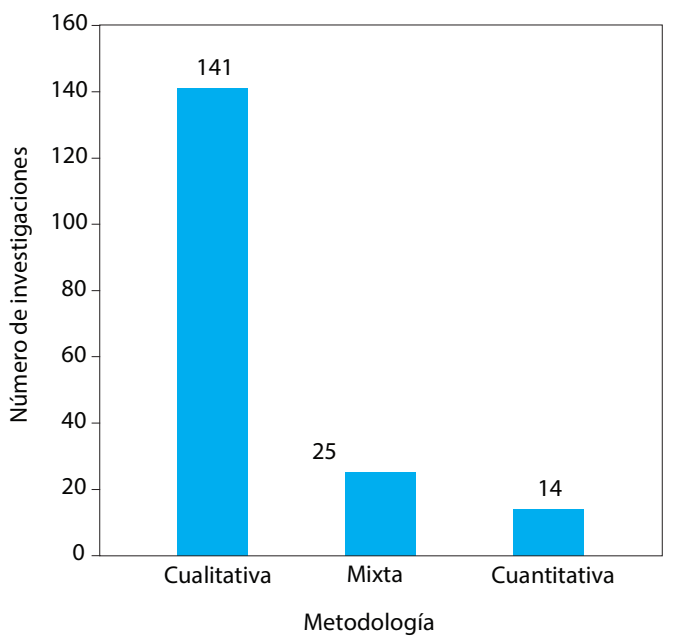

Fuente: elaboración propia con base en los datos de la Oficina Asesora de Planeación (ARN, 2019).
Como se observa en la figura 8, las investigaciones cualitativas tienen cierta permanencia y vigencia en el tiempo. Esto mismo no ocurre con las investigaciones cuantitativas, las cuales son más sensibles a factores que desconocemos, y que hacen que en algunos ańos sea poca la producción científica en materia de reintegración o reincorporación bajo este tipo de estrategias de análisis.

Los resultados de los enfoques de la investigación respecto a reintegración o reincorporación nos hacen sospechar que probablemente exista una gran cantidad y conocimiento detallado en esta materia. Las investigaciones cualitativas tienen como fin comprender la profundidad y el sentido de unos fenómenos sociales particulares. Sin embargo, esta gran

\section{FIGURA 8. FRECUENCIA DE LOS ENFOQUES METODOLÓGICOS}

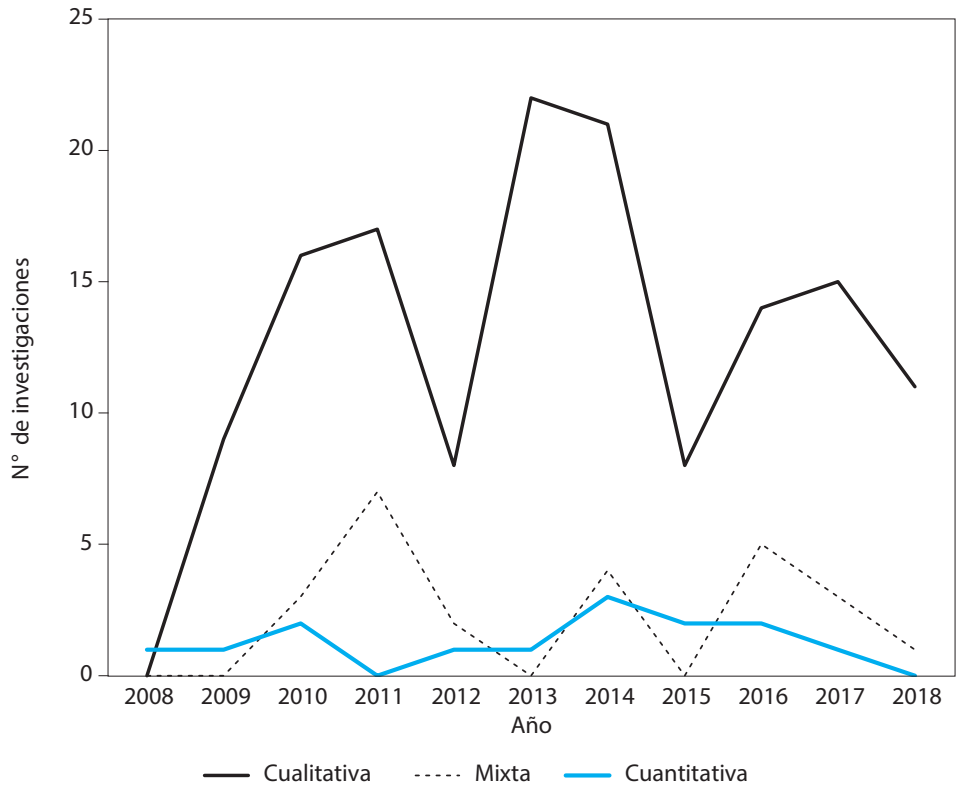

Fuente: elaboración propia con base en los datos de la Oficina Asesora de Planeación (ARN, 2019). 
cantidad de conocimiento cualitativo difícilmente puede ser comparable. Por ello, y al observar pocas investigaciones cuantitativas, cuyo fin es encontrar regularidades, comprobar hipótesis y comparar, sospechamos que falta, en el marco de las investigaciones científicas, impulsar más investigaciones con este enfoque.

Esto se corrobora con los hallazgos obtenidos al discriminar los instrumentos de recolección de información utilizados por los actores científicos. Como muestra la figura 9, la tendencia a realizar investigaciones cualitativas se refleja en la frecuencia de uso de técnicas como las entrevistas (abiertas o estructuradas), los grupos focales o la revisión documental. Pocas han utilizado las bases de datos disponibles en la ARN.

\section{Oportunidades y tensiones}

La consolidación de una gobernanza de la ciencia, en donde los científicos y las institu- ciones de gobierno trabajen articuladamente para construir un conocimiento que realmente incida en la formulación, ejecución, evaluación y el fortalecimiento de las políticas públicas requiere esfuerzos en doble vía. Para el caso de las instituciones de gobierno debe existir un compromiso explícito de movilizar los recursos humanos, financieros y tecnológicos que se requieran para poder interactuar con la ciencia.

Esto implica, más allá de un ejercicio discursivo, un esfuerzo por incluir la gestión del conocimiento en todos los niveles y procesos de la organización, es decir, lograr una efectiva inclusión en los planes y programas, en los presupuestos y en la conformación de equipos de trabajo dedicados exclusivamente a ese objeto. De no disponer de estas condiciones básicas, resulta muy difícil que las instituciones de gobierno logren procesos conjuntos de creación de conocimiento.

En este contexto, creemos quela ARN ha realizado importantes esfuerzos para transformar

FIGURA 9. INSTRUMENTOS DE RECOLECCIÓN DE INFORMACIÓN

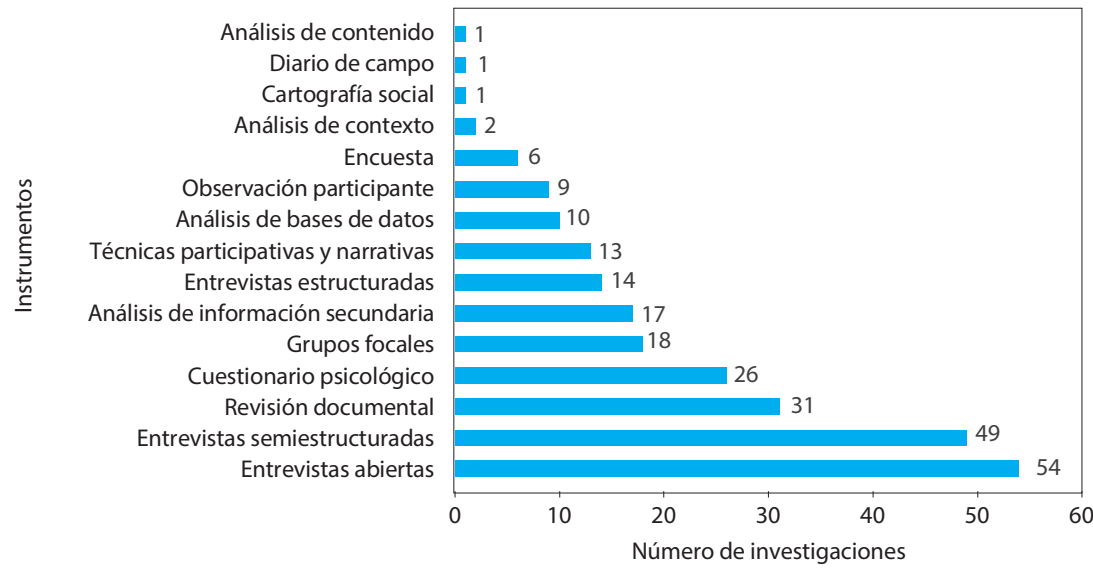

Fuente: elaboración propia con base en los datos de la Oficina Asesora de Planeación (ARN, 2019). 
la tradicional relación Estado-ciencia, en la cual generalmente solo se transfiere información como parte de un ejercicio de transparencia pública, a través de la creación de herramientas que permiten construir alianzas para trabajar en la generación de conocimiento sobre los procesos de reintegración y reincorporación de excombatientes. Esto sin duda representa una importante oportunidad.

Por otro lado, para que la articulación funcione, las personas encargadas de liderar los proyectos de ciencia e investigación deben comprometerse con crear metodologías y herramientas que no generen posibles afectaciones a la población que de forma voluntaria a decidido ayudarlos. Al respecto, funcionarios de la ARN consideran que los comités de ética de las universidades pueden jugar un rol fundamental en este aspecto.

De igual forma, la realización de ejercicios poco exhaustivos de revisión de literatura académica, que permitan determinar de forma concreta y acertada los vacíos de conocimiento que se pretenden trabajar, es uno de los factores por los cuales la ARN decide rechazar proyectos de ciencia e investigación. Este factor es importante, debido a que en un escenario de pocos y valiosos recursos, las instituciones de gobierno deben realizar ejercicios de priorización para evitar un uso inadecuado, lo cual se puede presentar al apoyar una investigación académica que arroje resultados que ya se encuentren descritos por otros autores.

Una situación que también genera tensión entre la ARN y la comunidad científica es la transferencia de conocimiento una vez finalizados los proyectos de ciencia e investigación. En la mayoría de las entrevistas realizadas se en- contró la preocupación por el bajo porcentaje de ejercicios de transferencia de conocimiento, lo cual impacta la motivación de los funcionarios al no poder ver los resultados del esfuerzo realizado para lograr el apoyo acordado.

Para la población excombatiente que participa de forma voluntaria en los proyectos de ciencia e investigación la situación es aún más preocupante, debido a que ante la imposibilidad de conocer los resultados del proyecto del que hicieron parte, se pueden generar sentimientos de rechazo ante la invitación de otros académicos, lo cual impacta profundamente a la ciencia, en tanto sus conocimientos $\mathrm{y}$ experiencias son fundamentales para lograr un fortalecimiento de las políticas de las cuales son beneficiarios.

Finalmente, con el fin de aportar elementos que puedan ser de utilidad para las personas interesadas en estudiar los temas de reintegración y reincorporación, consultamos a los funcionarios encargados de la coordinación de la política de la ARN en los territorios sobre los temas que consideran deben ser trabajados desde la ciencia. Al respecto se encontró consenso frente a los siguientes temas: reconciliación y convivencia en los territorios, sostenibilidad de proyectos productivos, estudios desde una perspectiva de enfoque de género y enfoques diferenciales, contexto familiar, asociación y cooperativismo entre poblaciones vulnerables y participación política de excombatientes.

Sumado a lo anterior, creemos que otro factor que debe ser tenido en cuenta tanto por la ARN como por la comunidad científica es la promoción de procesos de construcción de conocimiento desde las regiones, ya que esto permite una mayor sostenibilidad a las inves- 
tigaciones, así como una lectura y una apropiación mucho más acertada. Con la mirada regional, sin duda se realizarán importantes aportes a las políticas públicas de reintegración y reincorporación.

\section{CONCLUSIONES}

Este trabajo ha llevado a cabo una descripción de las tendencias de la investigación científica en materia de reintegración y reincorporación. Ha encontrado que existen algunos temas dominantes, como género, reintegración económica, percepciones sobre excombatientes y paz, reconciliación-convivencia y NNAJ. También, que existe una concentración de los estudios en algunas regiones de Colombia que no necesariamente han sido golpeadas por la violencia y el conflicto, como CundinamarcaBoyacá, Antioquia-Chocó, Valle del Cauca, Meta-Orinoquia y Santander.

En ese sentido, parece haber algunos intereses prácticos de los actores de ciencia e investigación que no necesariamente tienen que ver con las dinámicas de la violencia y la paz en los territorios. Por tanto, falta descentralizar la oferta científica para indagar acerca de los procesos de reintegración y reincorporación en las zonas más críticas.

Del mismo modo, nuestros hallazgos nos permiten observar que existe una preponderancia en enfocar los estudios en materia de reintegración y reincorporación a través de marcos analíticos cualitativos. Sospechamos que ello se debe a dos factores. El primero, al hecho de que refleja los enfoques y las estrategias de investigación que privilegian los científicos en el país; valdría la pena comparar estas cifras con los estudios en reintegración y reincorporación de los actores internacionales de ciencia. El segundo, que parece existir un desconocimiento de los datos y la información cuantitativa a través de los cuales es posible adelantar investigaciones bajo estos enfoques.

Por otra parte, los resultados sugieren que existe ya una comunidad especializada de actores de ciencia en materia de reintegración y reincorporación. Por lo cual, creemos que existen oportunidades de diálogo, cooperación y transferencia de conocimiento entre estos actores. Asimismo, con los datos, la información obtenida y los enfoques es posible construir marcos analíticos más amplios, que condensen teóricamente cómo se entiende la relación entre investigación, ciencia y gobierno en materia de reintegración y reincorporación, con sus tensiones y consensos.

\section{REFERENCIAS}

Agencia Colombiana para la Reintegración (ACR), Centro Mundial de Solución de Conflictos (CMSC) y Oficina del Alto Comisionado para la Paz (OACP) (2010). Desarme, desmovilización y reintegración DDR en Colombia: lecciones aprendidas y resultados del proceso 2002-2010. Bogotá: ACR, CMSC, OACP.

Agencia Colombiana para la Reintegración (ACR) y Organización Internacional para las Migraciones (OIM) (2013). First Global Disarmament, Demobilization and Reintegration (DDR) Summit: Global DDR Challenges and Techniques, South-South Cooperation, and Rural and Territorial Reintegration. Bogotá: ACR y OIM. 
Agencia Colombiana para la Reintegración (ACR) (2016). Estrategia de gestión del conocimiento. Bogotá: ACR. Agencia Colombiana para la Reintegración (ACR). (2016). Artículo 1. Resolución número 1356. Recuperado de www.reintegracion.gov.co/es/agencia/ Documentos\%20Resoluciones/Resolución\%20 1356.pdf

Agencia Colombiana para la Reintegración (ACR) (2016). Artículo 1. Resolución número 2687. Recuperado de www.reintegracion.gov.co/es/agencia/Documentos\%20Resoluciones/Resoluci\%C3\%B3n\%20 2687\%20de\%202016.pdf

Agencia para la Reincorporación y la Normalización (ARN) (2017). La reintegración en Colombia: construimos paz desde el territorio. Recuperado de reintegracion.gov.co/es/la-reintegracion/Cifras/ Hoja\%20de\%20datos\%20ARN\%20-\%20Septiembre\%20de\%202017.pdf

Agencia para la Reincorporación y la Normalización (ARN) (2017). La ACR fortalece su institucionalidad y pasa a ser la Agencia para la Reincorporación y la Normalización (ARN). Recuperado de reintegracion.gov.co/es/sala-de-prensa/noticias/ Paginas/2017/05/COMUNICADO-OFICIAL-ACRpasa-a-ser-ARN.aspx

Agencia para la Reincorporación y la Normalización (ARN) (2016). Informe 2016 Estrategia de Gestión del Conocimiento. Bogotá: ARN.

Amin, A. (1997). Beyond Market and Hierarchy: Interactive Governance and Social Complexity. Cheltenham: Elgar.

Beunen, R. y Opdam, P. (2011). When landscape planning becomes landscape governance, what happens to the science? Netherlands: Wageningen University.

Bora, A. y Hausendorf, H. (2006). Participatory science governance revisited normative expectations versus empirical evidence. Science and Public Policy, 33. https://doi.org/10.3152/147154306781778740.
Borzel, T. A. (1998). Organizing Babylon-on the different conceptions of policy networks. Public Administration, 76, 253-273.

Cárdenas J. y ACR (2015). First Global Disarmament, Demovilization and Reintegration (DDR) Summit. Bogotá: Agencia Colombiana para la Reintegración. Colombia. Recuperado de http://www.academia. edu/10480567/First_Global_Disarmament_Demobilization_and_Reintegration_DDR_Summit

Cárdenas, J. y Pérez, N. (2019). Dilemas en la implementación de un acuerdo de paz: “powersharing" en el proceso de reincorporación de las FARC. En Excombatientes y acuerdo de paz con las FARC-EP en Colombia: balance de la etapa temprana (pp. 69-85). Bogotá: Ediciones Uniandes.

CCDDR (2009). La contribución de Cartagena al desarme, la desmovilización y la reintegración (DDR). Cartagena: CCDDR.

Comisión Europea (2009). Gobernanza global de la ciencia. Informe del Grupo de expertos sobre gobernanza global de la ciencia a la Dirección de Ciencia, Economía y Sociedad Dirección General de Investigación. Recuperado de https://ec.europa. eu/research/science-society/document_library/ pdf_06/global-governance-290909_es.pdf

Cooke, P. (2002). Regional innovation systems: General findings and some new evidence from biotechnology clusters. Journal of Technology Transfer, 27(1), 133-145.

Geuna, A. y Muscio A. (2009). The Governance of University Knowledge Transfer: A Critical Review of the Literature. Minerva 47(1), 93-114.

Kuhlmann, S. (2007). Governance of innovation: practice, policy and theory as dancing partners. Enschede: University of Twente.

Knorr, C. (1999). Epistemic Cultures: How the Sciences Make Knowledge. Cambridge: Harvard University Press. 
Kooiman, J. (2003). Governing as Governance. London: Sage.

Lehoux, P., Daudelin, G., Lavis, Denis, J. L., Abelson, J. y Miller, F. A. (2010). Exploring the conundrum of the new knowledge production regime: An ethnographic case study on the governance and outcomes of a science/policy network in genetics. Science and Public Policy, 37(10), 737-750.

López - Roldán, P. y Fachelli, S. (2015). Análisis descriptivo de datos con una variable. En R. P. López y
S. Fachelli, Metodología de la Investigación Social Cuantitativa. Barcelona: Universitat Autónoma de Barcelona.

Lyall, C. (2007). Changing boundaries: the role of policy networks in the multi-level governance of science and innovation in Scotland. Edinburgh: University of Edinburgh. 